\title{
The effects of quadrivalent human papillomavirus vaccination in recurrent respiratory papillomatosis (RRP)
}

\author{
Jack Gao, Ali Al-Lami, Elfy Chevretton
}

jack7.gao7@gmail.com; aklami@doctors.org.uk; Elfy.Chevretton@gstt.nhs.uk

\section{Introduction}

Recurrent respiratory papillomatosis (RRP) is a benign neoplasm of the respiratory tract involving the formation of laryngeal papillomata ${ }^{1}$. Frequent surgical resection is required in order to maintain airway patency and improve voice quality. Papillomata are associated with benign human papillomavirus (HPV) subtypes -6 and $-11^{2}$ and, hence, the quadrivalent HPV vaccine (Gardasil ${ }^{\circledR}$ ) has been proposed as an adjuvant therapy ${ }^{3}$ to prevent new papilloma formation at additional sites. There is currently a relative lack of data for the efficacy of the vaccine in this role

\section{Aims}

In this case series, we aim to observe the effects of therapeutic HPV vaccination with Gardasil ${ }^{\circledR}$ on the frequency of surgical procedures in patients with RRP.

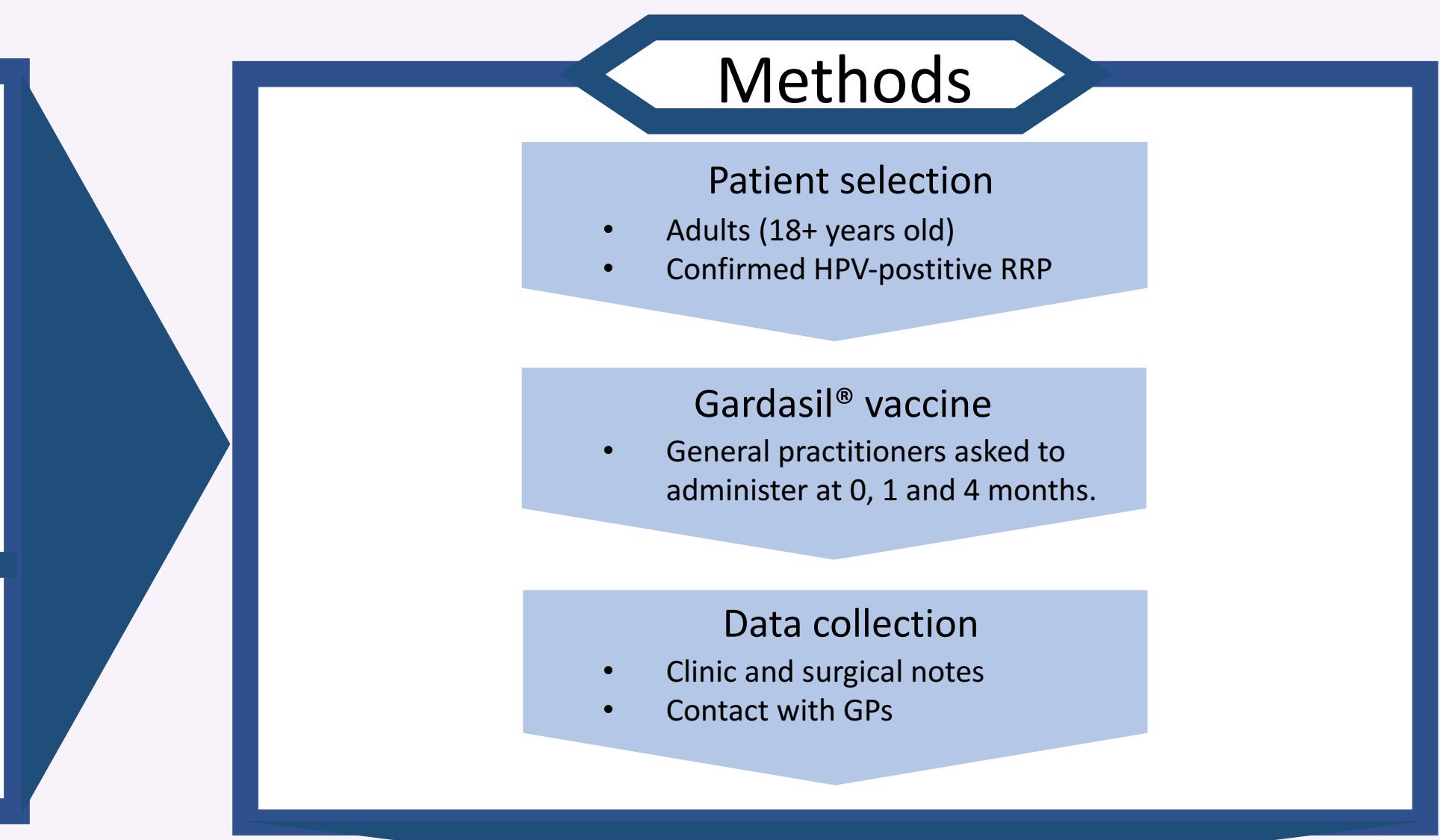

Results

\section{Patients identified}

\begin{tabular}{|c|c|c|c|c|c|c|c|c|c|c|c|c|c|c|c|}
\hline \multirow[b]{2}{*}{ Patient } & \multirow[b]{2}{*}{$\begin{array}{l}\text { Gender } \\
(\mathrm{M} / \mathrm{F})\end{array}$} & \multirow[b]{2}{*}{$\begin{array}{c}\text { Age of Diagnosis } \\
\text { (years) }\end{array}$} & \multirow[b]{2}{*}{$\begin{array}{l}\text { Juvenile/Adult } \\
\text {-onset }\end{array}$} & \multirow[b]{2}{*}{$\begin{array}{l}\text { Presenting } \\
\text { Complaint }\end{array}$} & \multirow[b]{2}{*}{$\begin{array}{l}\text { Extent of } \\
\text { papillomata }\end{array}$} & \multicolumn{2}{|c|}{ Surgical modalities } & \multirow[b]{2}{*}{$\begin{array}{l}\text { Vaccination } \\
\text { Age (years) }\end{array}$} & \multirow[b]{2}{*}{ Adjuvants } & \multicolumn{3}{|c|}{ Pre-vaccination } & \multicolumn{3}{|c|}{ Post-vaccination } \\
\hline & & & & & & $\begin{array}{l}\text { Helica } \\
\text { cautery }\end{array}$ & Microdebrider & & & $\begin{array}{c}\text { Follow-up period } \\
\text { (years) }\end{array}$ & $\begin{array}{l}\text { No. of surgical } \\
\text { procedures }\end{array}$ & $\begin{array}{c}\text { Procedures/ } \\
\text { year }\end{array}$ & $\begin{array}{c}\text { Follow-up period } \\
\text { (years) }\end{array}$ & $\begin{array}{l}\text { No. of surgical } \\
\text { procedures }\end{array}$ & $\begin{array}{l}\text { Procedures } \\
\text { /year }\end{array}$ \\
\hline 1 & $M$ & 24 & AoRRP & Hoarseness & Vocal cords & $x$ & & 25 & None & 1.76 & 2 & 1.14 & 0.11 & 0 & 0.00 \\
\hline 2 & $\mathrm{~F}$ & 64 & AoRRP & Hoarseness & $\begin{array}{l}\text { Vocal cords } \\
\text { Left arytenoid and }\end{array}$ & $x$ & & 65 & None & 5.33 & 2 & 0.38 & 1.57 & 0 & 0.00 \\
\hline 3 & $\mathrm{~F}$ & $<31$ & AoRRP & Hoarseness & $\begin{array}{l}\text { vocal cord } \\
\text { Vocal cords to }\end{array}$ & $x$ & $x$ & 45 & None under this clinic & 14.10 & 4 & 0.28 & 3.11 & 0 & 0.00 \\
\hline 4 & M & 7 & JoRRP & Hoarseness & trachea & & $x$ & 34 & None under this clinic & 14.72 & 12 & 0.82 & 3.12 & 2 & 0.64 \\
\hline 5 & M & 26 & AoRRP & Hoarseness & $\begin{array}{l}\text { Vocal cords } \\
\text { Vocal cords and }\end{array}$ & $x$ & $x$ & 34 & None under this clinic & 6.40 & 8 & 1.25 & 1.47 & 1 & 0.68 \\
\hline 6 & $\mathrm{~F}$ & 26 & AORRP & Hoarseness & supraglottis & & $x$ & 26 & None & 1.00 & 2 & 2.00 & 0.53 & 0 & 0.00 \\
\hline
\end{tabular}

Average frequency of procedures pre- and post- Gardasil
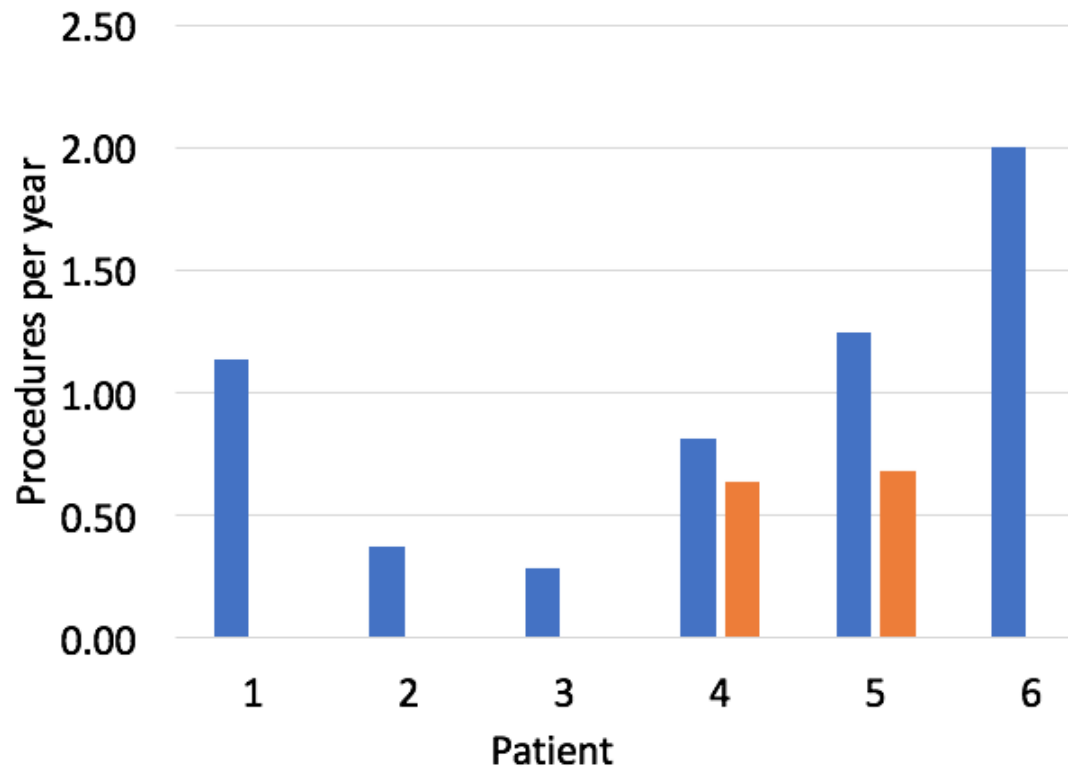

- Pre-Gardasil a Post-Gardasil
Procedures by year pre- and post- Gardasil

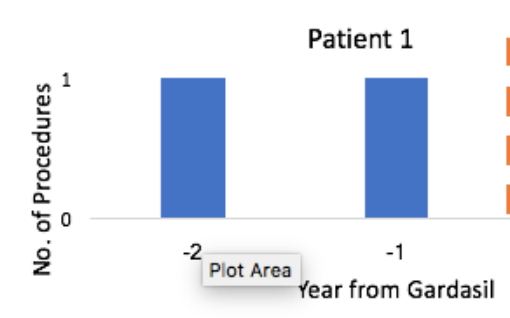

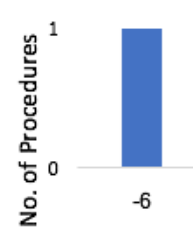
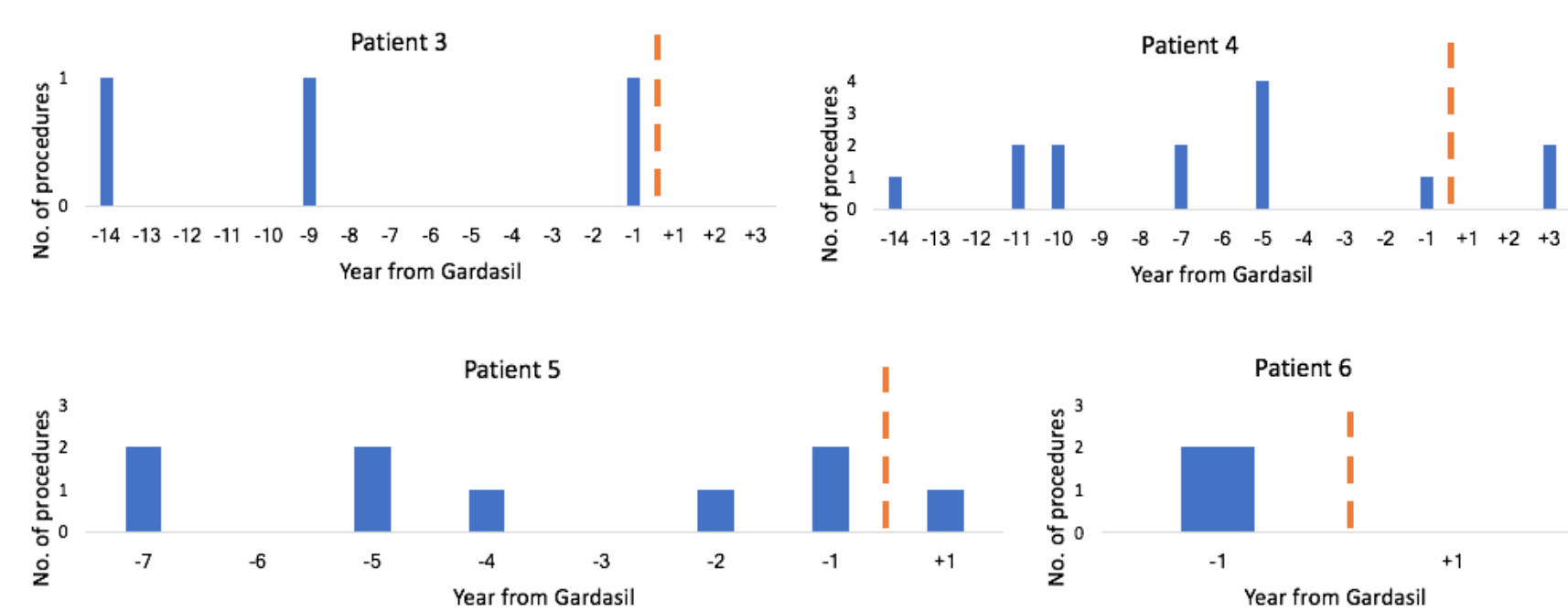

\section{Discussion/Summary}

Literature review

Juvenile-onset RRP - 5 case reports: 3 showed complete remission within follow-up periods $5,7,8 ; 2$ showed increased inter-surgical interval $\left.\right|^{4,9}$. One uncontrolled interv
clinical course in the year before and after Gardasil ${ }^{6} 6$ $85 \%$ without recurrence at 2 years follow up.

Results summary

no further procedures after Gardasite.

2 patients had fewer procedures after Gardasil

Limitations

Small number of patients

Relatively short follow-up period 